Commun. Korean Math. Soc. 28 (2013), No. 3, pp. 487-500

http://dx.doi.org/10.4134/CKMS.2013.28.3.487

\title{
CONTINUITY OF APPROXIMATE POINT SPECTRUM ON THE ALGEBRA $B(X)$
}

\author{
Salvador Sánchez-Perales and Victor A. Cruz-Barriguete
}

\begin{abstract}
In this paper we provide a brief introduction to the continuity of approximate point spectrum on the algebra $B(X)$, using basic properties of Fredholm operators and the SVEP condition. Also, we give an example showing that in general it not holds that if the spectrum is continuous an operator $T$, then for each $\lambda \in \sigma_{s-F}(T) \backslash \overline{\rho_{s-F}^{ \pm}(T)}$ and $\epsilon>0$, the ball $B(\lambda, \epsilon)$ contains a component of $\sigma_{s-F}(T)$, contrary to what has been announced in [J. B. Conway and B. B. Morrel, Operators that are points of spectral continuity II, Integral Equations Operator Theory 4 (1981), 459-503] page 462.
\end{abstract}

\section{Introduction}

It is known that the properties of the spectrum of a linear operator defines its behavior. Since exact computations of the spectrum are almost always impossible, we attempt obtain it in approximate form, so the study of the continuity of the spectrum or any of its parts takes an essential role.

Newburgh (see [14]) was one of the first to make a systematic investigation about the continuity of spectrum. After him several authors have researched the subject extensively (for example [3], [4], [6], [8], [9], [10] and [11]).

Conway and Morrel [7] characterize the continuity of the approximate point spectrum in the class of bounded linear operators defined on Hilbert spaces. In this paper we observe that for the sufficient condition in that characterization the several hypotheses can be reduced on the class of bounded linear operators (now defined on Banach spaces) whose adjoint has SVEP. Also, we give an example showing that the proof of their characterization mentioned has a small omission.

Received May 19, 2012

2010 Mathematics Subject Classification. Primary 47A10, 47A53.

Key words and phrases. approximate point spectrum, continuity of the spectrum. 


\section{The spectrum of a bounded operator}

Let $X$ and $Y$ be normed spaces. A linear operator $T: X \rightarrow Y$ is bounded, if there exists a constant $M \geq 0$ such that

$$
\|T x\|_{Y} \leq M\|x\|_{X} \quad \text { for all } x \in X .
$$

It is easy to see that bounded linear operators are continuous and even uniformly continuous whit respect to their norms. Conversely, a linear operator is bounded if it is continuous at 0 .

If $T: X \rightarrow Y$ is a bounded linear operator, then its norm $\|T\|$ is defined by

$$
\|T\|=\inf \left\{\beta \geq 0:\|T x\|_{Y} \leq \beta\|x\|_{X} \text { for all } x \in X\right\} .
$$

Equivalent expressions of $\|T\|$ are:

$$
\|T\|=\sup _{x \neq 0} \frac{\|T x\|_{Y}}{\|x\|_{X}} ; \quad\|T\|=\sup _{\|x\|_{X} \leq 1}\|T x\|_{Y} ; \quad\|T\|=\sup _{\|x\|_{X}=1}\|T x\|_{Y} .
$$

Let $X$ and $Y$ be complex Banach spaces and let $B(X, Y)$ denote the space of all bounded linear operators from $X$ to $Y$, abbreviate $B(X, X)$ to $B(X)$. This set with the usual operations of addition and multiplication by a scalar is a vector space over $\mathbb{C}$, moreover, $B(X)$ is an algebra with the multiplication given by $(T, S) \mapsto T S$ (composition of functions). It is easy to see that $\|\cdot\|$, exposed in (2.1), defines a norm on $B(X)$ and satisfies the conditions: $\|T S\| \leq\|T\|\|S\|$, $T, S \in B(X)$, and $\|I\|=1$. Thus $B(X)$ is a Banach algebra with identity.

A class of special interest in $\mathcal{B}(X)$ are the compact operators, denoted by $\mathcal{K}(X)$. Remember that an operator $T \in \mathcal{B}(X)$ is compact if and only if for each bounded sequence $\left\{x_{n}\right\}_{n \in \mathbb{N}}$ in $X$, there exists a subsequence $\left\{x_{n_{k}}\right\}_{k \in \mathbb{N}}$ and an element $x \in X$ such that $T x_{n_{k}} \rightarrow x$. It is well known that $\mathcal{K}(X)$ is two-side ideal in $\mathcal{B}(X)$. This fact enables us to define the Calkin algebra over $X$ as the quotient algebra $C(X)=\mathcal{B}(X) / \mathcal{K}(X)$. Moreover, $\mathcal{K}(X)$ is a closed subspace of $\mathcal{B}(X)$, thus $C(X)$ with the norm $\|T+\mathcal{K}(X)\|=\inf _{U \in \mathcal{K}(X)}\|T+U\|$ is a Banach algebra. We shall use $\pi$ to denote the natural homomorphism of $\mathcal{B}(X)$ onto $C(X) ; \pi(T)=T+\mathcal{K}(X)$.

Since $B(X)$ is an algebra with identity we can consider, for an operator $T \in B(X)$, the set

$$
\rho(T)=\{\lambda \in \mathbb{C} \mid \lambda I-T \text { is invertible in } B(X)\} .
$$

This set is called the resolvent of $T$. Its complement, a more interested set, is denoted by $\sigma(T)$ and is called the spectrum of $T$. Namely,

$$
\sigma(T)=\{\lambda \in \mathbb{C} \mid \lambda I-T \text { is not invertible in } B(X)\} .
$$

It is well known that if $X$ is a finite-dimensional normed vector space and $T: X \rightarrow X$ is a linear operator, then the spectrum of $T$ is precisely the set of eigenvalues of $T$. Remember, a complex number $\lambda \in \mathbb{C}$, is an eigenvalue of an linear operator $T$, if there exists $x \in X$ with $x \neq 0$ such that $T x=\lambda x$. The set of all eigenvalues of $T$ is denoted by $\sigma_{p}(T)$. 
In general, for $X$ a complex Banach space, the spectrum of bounded linear operator $T \in B(X), \sigma(T)$, is a compact nonempty subset of $\mathbb{C}$. Further, this set is bounded by $\|T\|$, i.e., $\sigma(T) \subseteq B(0,\|T\|)$.

Example 2.1. We give the spectrum of some operators:

(a) Let $T: L^{2}[0,1] \rightarrow L^{2}[0,1]$ be defined by $(T f)(t)=t f(t)$. Then $\sigma(T)=$ $[0,1]$.

(b) Consider $l^{2}(N)=\left\{\left.\left\{x_{n}\right\}_{n \in \mathbb{N}} \subseteq \mathbb{C}\left|\sum_{n \in \mathbb{N}}\right| x_{n}\right|^{2}<\infty\right\}$. This is a Hilbert space with the inner product $\left\langle\left\{x_{n}\right\} ;\left\{y_{n}\right\}\right\rangle=\sum_{n \in \mathbb{N}} x_{n} \overline{y_{n}}$. Let $U: l^{2}(N) \rightarrow$ $l^{2}(N)$ be defined by $U(x)=\left(0, x_{1}, x_{2}, x_{3}, \ldots\right), x=\left(x_{1}, x_{2}, x_{3}, \ldots\right)$. Usually this operator is called the unilateral shift and its spectrum is $\sigma(U)=\{\lambda \in$ $\mathbb{C}|| \lambda \mid \leq 1\}$.

(c) Two operators $T, S \in B(X)$ are similar, if there exists $U \in B(X)$ invertible such that $U T=S U$. If $T$ and $S$ are similar, then $\sigma(T)=\sigma(S)$.

(d) Let $H$ be a complex Hilbert space. An operator $T \in B(H)$ is unitary if and only if $T^{*} T=T T^{*}=I$, where $T^{*}$ (the adjoint of $T$ ) is the unique operator $T^{*}: H \rightarrow H$ such that $\langle T x ; y\rangle=\left\langle x ; T^{*} y\right\rangle$ for all $x, y \in H$. If $T \in B(H)$ is unitary, then $\sigma(T)=\{\lambda \in \mathbb{C}|| \lambda \mid=1\}$.

The spectrum of a bounded linear operator can be divided into subsets of different ways, and each of these subsets are called parts of the spectrum. For $T \in B(X)$, the surjective spectrum and the approximate point spectrum of $T$ is defined, respectively, as

$$
\sigma_{s u}(T)=\{\lambda \in \mathbb{C} \mid \lambda I-T \text { is not surjective }\}
$$

and

$$
\sigma_{a p}(T)=\{\lambda \in \mathbb{C} \mid \lambda I-T \text { is not bounded below }\},
$$

where, remember an operator $T$ is bounded below if and only if there is $K>0$ such that $K\|x\| \leq\|T x\|$ for all $x \in X$. From this, it follows that $\lambda \in \sigma_{a p}(T)$ if and only if there is a sequence $\left\{x_{n}\right\}_{n \in \mathbb{N}}$ in $X$ with $\left\|x_{n}\right\|=1$ such that $(\lambda I-T) x_{n} \rightarrow 0$. Using this equivalence it is proved that $\sigma_{a p}(T)$ is a closed nonempty subset of $\mathbb{C}$, moreover, $\partial \sigma(T) \subseteq \sigma_{a p}(T)$.

Let $X$ and $Y$ be complex Banach spaces and consider $T \in B(X, Y)$. Denote $X^{*}$ (resp. $\left.Y^{*}\right)$ the dual of $X$ (resp. $Y$ ), i.e., $X^{*}=\mathcal{B}(X, \mathbb{C})$ and $Y^{*}=\mathcal{B}(Y, \mathbb{C})$. For $T \in \mathcal{B}(X, Y)$, the adjoint operator $T^{*}: Y^{*} \rightarrow X^{*}$ of $T$ is defined as:

$$
\left(T^{*} f^{*}\right)(x)=f^{*}(T x)
$$

for all $f^{*} \in Y^{*}$ and $x \in X$.

This definition is different to that given in Hilbert spaces, but it is a generalization. The adjoint $T^{*}$ of $T$ is a linear operator and $\left\|T^{*}\right\|=\|T\|$. Let $T, S \in \mathcal{B}(X, Y), A \in B(Y, Z)$ and $\gamma_{1}, \gamma_{2} \in \mathbb{C}$, the adjoint has the following easy properties:

$$
\left(\gamma_{1} T+\gamma_{2} S\right)^{*}=\gamma_{1} T^{*}+\gamma_{2} S^{*} \quad \text { and } \quad(A T)^{*}=T^{*} A^{*}
$$


Moreover, for $T \in B(X)$,

$$
\sigma(T)=\sigma\left(T^{*}\right), \quad \sigma_{a p}(T)=\sigma_{s u}\left(T^{*}\right) \text { and } \sigma_{a p}\left(T^{*}\right)=\sigma_{s u}(T) .
$$

\section{Parts of the spectrum given by semi-Fredholm operators}

Each linear operator $T: X \rightarrow X$ has associated two basic subspaces, its kernel $N(T)=\{x \in X \mid T x=0\}$ and its range $R(T)=\{T x \mid x \in X\}$. If $T$ is bounded its kernel $N(T)$ is a closed subspace, however, its range may be not closed.

Let $T: X \rightarrow X$ be a bounded operator. Define $\alpha(T)=\operatorname{dim} N(T)$ and $\beta(T)=\operatorname{dim} X / R(T)$, when theses spaces are finite dimensional, otherwise we set $\alpha(T)=\infty$ and $\beta(T)=\infty$. An operator $T \in B(X)$ is called upper semiFredholm (respectively lower semi-Fredholm) if it has closed range and $\alpha(T)<$ $\infty$ (respectively $\beta(T)<\infty$ ). The set of all upper (resp. lower) semi-Fredholm operator in $B(X)$ is denoted by $\Phi_{+}(X)$ (resp. $\Phi_{-}(X)$ ). We say that $T \in B(X)$ is a semi-Fredholm operator if $T \in \Phi_{+}(X) \cup \Phi_{-}(X)=\Phi_{ \pm}(X)$. The index of a semi-Fredholm operator $T$ is defined as $i(T)=\alpha(T)-\beta(T)$. When $T$ is both upper and lower semi-Fredholm, $T$ is said to be a Fredholm operator. This class of operators is denoted by $\Phi(X)$.

Remark 3.1. We highlight below some basic properties concerning algebraic and topological nature of the class of semi-Fredholm operators.

(a) If $T \in \Phi_{+}(X)$ and $S \in \Phi_{+}(X)$, then $T S \in \Phi_{+}(X)$. Analogously, if $U \in$ $\Phi_{-}(X)$ and $V \in \Phi_{-}(X)$, then $U V \in \Phi_{-}(X)$. Moreover, if $T \in \Phi(X)$ and $S \in \Phi(X)$, then $T S \in \Phi(X)$ and $i(T S)=i(T)+i(S)$.

(b) The sets $\Phi_{+}(X)$ and $\Phi_{-}(X)$ are mutually dual:

$$
T \in \Phi_{+}(X) \Leftrightarrow T^{*} \in \Phi_{-}\left(X^{*}\right)
$$

and

Moreover,

$$
T \in \Phi_{-}(X) \Leftrightarrow T^{*} \in \Phi_{+}\left(X^{*}\right)
$$

$$
\alpha\left(T^{*}\right)=\beta(T), \quad \alpha(T)=\beta\left(T^{*}\right) \text { and } \alpha(T)=\alpha\left(T^{* *}\right) .
$$

(c) Let $T \in \Phi_{+}(X)$. Then there exists $\epsilon>0$ such that $S \in B(X)$ and $\|S\|<\epsilon$ implies $T+S \in \Phi_{+}(X)$. Furthermore

$$
\alpha(T+S) \leq \alpha(T) \text { and } i(T+S)=i(T) .
$$

Analogously, if $T \in \Phi_{-}(X)$, then there exists $\epsilon>0$ such that for every $S \in B(X)$ with $\|S\|<\epsilon$ we have $T+S \in \Phi_{-}(X)$ and

$$
\beta(T+S) \leq \beta(T) \text { and } i(T+S)=i(T) .
$$

From this it follows that $\Phi_{+}(X)$ and $\Phi_{-}(X)$ are open subsets of $B(X)$ and the index function

$$
i: \Phi_{ \pm}(X) \rightarrow \mathbb{Z} \cup\{ \pm \infty\}
$$

is continuous. 
(d) Let $T \in B(X)$. Then, there exist $V \in B(X)$ and $K \in K(X)$ such that $V T=I+K$ if and only if $T \in \Phi_{+}(X)$ and there exists a bounded projection of $X$ onto $R(T)$.

Also, there exist $U \in B(X)$ and $K \in K(X)$ such that $T U=I+K$ if and only if $T \in \Phi_{-}(X)$ and there exists a bounded projection of $X$ onto $N(T)$.

The proof theses important results may be found in Caradus, Pfaffenberger, and Yood [5].

Let $M$ be a closed subspace of a Banach space $X$. If there exists a closed subspace $N$ of $X$ such that $X=M \oplus N$, then $M$ is said to be complemented in $X$. Equivalently, $M$ is complemented in $X$ if and only if there exists $P$ a bounded projection of $X$ onto $M$.

Let $Z$ be a Banach algebra with identity. Denote by $G_{l}(Z)\left(\right.$ resp. $\left.G_{r}(Z)\right)$ the set of all left (resp. right) invertible elements of $Z$. Using Remark 3.1(d), we obtain the next proposition.

Proposition 3.2. Let $\pi: B(X) \rightarrow C(X)$ be the canonic homomorphism. Then for $T \in B(X)$ the following equivalences holds.

(a) $\lambda I-\pi(T) \in G_{l}(C(X)) \Leftrightarrow \lambda I-T \in \Phi_{+}(X)$ and $R(\lambda-T)$ is complemented in $X$

(b) $\lambda I-\pi(T) \in G_{r}(C(X)) \Leftrightarrow \lambda I-T \in \Phi_{-}(X)$ and $N(\lambda-T)$ is complemented in $X$.

For $T \in \mathcal{B}(X)$ and $k \in \mathbb{N} \cup\{-\infty, \infty\}$, let $\rho_{s-F}^{k}(T)$ denote the set of $\lambda \in \mathbb{C}$ for which $\lambda I-T \in \Phi_{ \pm}(X)$ and $i(\lambda I-T)=k$. Put $\rho_{s-F}^{+}(T)=\underset{1 \leq k \leq \infty}{\cup} \rho_{s-F}^{k}(T)$, $\rho_{s-F}^{-}(T)=\bigcup_{-\infty \leq k \leq-1} \rho_{s-F}^{k}(T)$ and $\rho_{s-F}^{ \pm}(T)=\rho_{s-F}^{-}(T) \cup \rho_{s-F}^{+}(T)$. Also, define the sets:

$$
\begin{aligned}
\phi_{+}(T) & =\left\{\lambda \in \rho_{s-F}^{+}(T) \mid N(\lambda I-T) \text { is complemented in } X\right\}, \\
\phi_{+\infty}(T) & =\left\{\lambda \in \rho_{s-F}^{+\infty}(T) \mid N(\lambda I-T) \text { is complemented in } X\right\}, \\
\phi_{-}(T) & =\left\{\lambda \in \rho_{s-F}^{-}(T) \mid R(\lambda I-T) \text { is complemented in } X\right\}, \\
\phi_{-\infty}(T) & =\left\{\lambda \in \rho_{s-F}^{-\infty}(T) \mid R(\lambda I-T) \text { is complemented in } X\right\} .
\end{aligned}
$$

We set $\phi_{ \pm \infty}(T)=\phi_{-\infty}(T) \cup \phi_{+\infty}(T)$. One can prove that $\phi_{+}(T), \phi_{-}(T)$, $\phi_{+\infty}(T)$ and $\phi_{-\infty}(T)$ are open subsets of $\mathbb{C}$ and that in Hilbert spaces the equalities $\phi_{+}(T)=\rho_{s-F}^{+}(T), \phi_{+\infty}(T)=\rho_{s-F}^{+\infty}(T), \phi_{-}(T)=\rho_{s-F}^{-}(T)$ and $\phi_{-\infty}(T)=\rho_{s-F}^{-\infty}(T)$ holds.

All classes of operators mentioned above motivate the study of some other distinguished parts of the spectrum: The Fredholm spectrum of an operator $T \in B(X)$ is defined as $\sigma_{e}(T)=\{\lambda \in \mathbb{C} \mid \lambda I-T \notin \Phi(X)\}$, the semi-Fredholm spectrum of $T$ is $\sigma_{s-F}(T)=\left\{\lambda \in \mathbb{C} \mid \lambda I-T \notin \Phi_{ \pm}(X)\right\}$, the Weyl spectrum of $T$ is $\sigma_{W}(T)=\{\lambda \in \mathbb{C} \mid \lambda I-T \notin \Phi(X)$ or $i(\lambda I-T) \neq 0\}$. The left essential spectrum (resp. the right essential spectrum) of $T \in \mathcal{B}(X)$, are defined 
respectively by $\sigma_{l e}(T)=\left\{\lambda \in \mathbb{C} \mid \lambda I-\pi(T) \notin G_{l}(C(X))\right\}$ and $\sigma_{r e}(T)=\{\lambda \in$ $\left.\mathbb{C} \mid \lambda I-\pi(T) \notin G_{r}(C(X))\right\}$. Finally put

$$
\sigma_{l r e}(T)=\sigma_{l e}(T) \cap \sigma_{r e}(T) .
$$

It is clear that

$$
\sigma_{s-F}(T) \subseteq \sigma_{\text {lre }}(T),
$$

but the opposite inclusion is not always satisfied in general Banach spaces. However when $T$ is defined on a Hilbert space $H$, then $\sigma_{s-F}(T)=\sigma_{\text {lre }}(T)$ is satisfied, because every closed subspace of $H$ is complemented in $H$.

Proposition 3.3. If $T \in \mathcal{B}(X)$, then

(a) $\sigma_{e}(T)=\sigma_{s-F}(T) \cup \rho_{s-F}^{ \pm \infty}(T)$;

(b) $\sigma_{e}(T)=\sigma_{l r e}(T) \cup \phi_{ \pm \infty}(T)$;

(c) $\partial \sigma_{e}(T) \subseteq \sigma_{s-F}(T)$.

Proof. (a) It is trivial. (b). Take $\lambda \in \sigma_{e}(T)$. If $\lambda \in \sigma_{l e}(T)$ and $\lambda \in \sigma_{r e}(T)$, then $\lambda \in \sigma_{\text {lre }}(T)$. Suppose that $\lambda I-\pi(T)$ is left invertible in $C(X)$, then by Proposition 3.2 (a), $\lambda I-T \in \Phi_{+}(X)$ and $R(\lambda I-T)$ is complemented in $X$. From $\lambda \in \sigma_{e}(T)$, we obtain that $\beta(\lambda I-T)=\infty$, thus $i(\lambda I-T)=-\infty$ and hence $\lambda \in \phi_{-\infty}(T)$. A similar argument shows that if $\lambda I-\pi(T)$ is right invertible in $C(X)$, then $\lambda \in \phi_{+\infty}(T)$. Consequently, $\sigma_{e}(T) \subseteq \sigma_{\text {lre }}(T) \cup \phi_{ \pm \infty}(T)$. The opposite inclusion is obtained readily.

(c) Note that $\rho_{s-F}^{ \pm \infty}(T) \subseteq \operatorname{int} \sigma_{e}(T)$, and this implies that

$$
\partial \sigma_{e}(T)=\overline{\sigma_{e}(T)} \backslash \operatorname{int} \sigma_{e}(T) \subseteq \sigma_{e}(T) \backslash \rho_{s-F}^{ \pm \infty}(T) .
$$

Then, by item $(a), \partial \sigma_{e}(T) \subseteq \sigma_{s-F}(T)$.

The next theorem establishes a relationship of connectedness between $\sigma_{l r e}(T)$ and $\sigma_{e}(T)$.

Theorem 3.4. Let $T \in \mathcal{B}(X)$. If $C$ is a component of $\sigma_{\text {lre }}(T)$ and $C \cap$ $\overline{\phi_{ \pm \infty}(T)}=\emptyset$, then $C$ is a component of $\sigma_{e}(T)$.

Proof. From Proposition 3.3(b),

$$
\sigma_{e}(T)=\left[\sigma_{l r e}(T) \backslash \partial \phi_{ \pm \infty}(T)\right] \cup \overline{\phi_{ \pm \infty}(T)} .
$$

We claim that

$$
\left[\sigma_{\text {lre }}(T) \backslash \partial \phi_{ \pm \infty}(T)\right] \cap \overline{\phi_{ \pm \infty}(T)}=\emptyset .
$$

Assume otherwise, then there exists $\lambda \in\left[\sigma_{l r e}(T) \backslash \partial \phi_{ \pm \infty}(T)\right] \cap \overline{\phi_{ \pm \infty}(T)}$. This implies, since $\overline{\phi_{ \pm \infty}(T)}=\phi_{ \pm \infty}(T) \cup \partial \phi_{ \pm \infty}(T)$, that $\lambda \in \phi_{ \pm \infty}(T)$. If $\lambda \in \phi_{+\infty}(T)$, then $\lambda I-T \in \Phi_{-}(X)$ and $N(\lambda I-T)$ is complemented in $X$, so by Proposition 3.2(b), $\lambda I-\pi(T)$ is right invertible in $C(X)$, this contradicts that $\lambda \in \sigma_{\text {lre }}(T)$. When $\lambda \in \Phi_{-\infty}(T)$ also we have a contradiction. Therefore the equality (3.1) follows. 
Let $D$ be a connected subset of $\sigma_{e}(T)$ such that $C \subseteq D$. Then

$$
D=\left[D \cap\left(\sigma_{\text {lre }}(T) \backslash \partial \phi_{ \pm \infty}(T)\right)\right] \cup\left[D \cap \overline{\phi_{ \pm \infty}(T)}\right] .
$$

We set $E=D \cap\left(\sigma_{\text {lre }}(T) \backslash \partial \phi_{ \pm \infty}(T)\right)$. Since $C \cap \overline{\phi_{ \pm \infty}(T)}=\emptyset$, we obtain that $C \subseteq E$, and this implies that either $E=C$ or $E$ is not connected. If $E$ fails to be connected, then there exist $E_{1}$ and $E_{2}$ compact subsets of $\sigma_{\text {lre }}(T)$ such that $E=E_{1} \cup E_{2}, E_{1} \cap E_{2}=\emptyset, E_{1} \neq \emptyset$ and $E_{2} \neq \emptyset$. Observe that

$$
D=E_{1} \cup\left[E_{2} \cup\left(D \cap \overline{\phi_{ \pm \infty}(T)}\right)\right],
$$

and by (3.1),

$$
\overline{E_{1}} \cap\left[E_{2} \cup\left(D \cap \overline{\phi_{ \pm \infty}(T)}\right)\right]=\emptyset \text { and } E_{1} \cap \overline{E_{2} \cup\left(D \cap \overline{\phi_{ \pm \infty}(T)}\right)}=\emptyset .
$$

Consequently $D$ is not connected, which is a contradiction. Thus $E=C$. Therefore

$$
D=C \cup\left(D \cap \overline{\phi_{ \pm \infty}(T)}\right) .
$$

Again, from $C \cap \overline{\phi_{ \pm \infty}(T)}=\emptyset$ and since $D$ is connected, we have $D \cap$ $\overline{\phi_{ \pm \infty}(T)}=\emptyset$. Therefore, $C=D$. We conclude that $C$ is a component of $\sigma_{e}(T)$.

\section{Spectral continuity}

Let $\mathcal{S}$ denote the collection of all non-empty compact sets of $\mathbb{C}$. The spectrum, the approximate point spectrum and the surjective spectrum can be viewed as functions from $B(X)$ to $\mathcal{S}$ mapping operators $T \in B(X)$ into their respective spectra. We study the continuity of these functions, using uniform norm in $B(X)$ and Hausdorff metric in $\mathcal{S}$. It is well known that the convergence in $\mathcal{S}$ with the Hausdorff metric can be characterized through the concepts of limit inferior and superior.

Let $\left\{E_{n}\right\}_{n \in \mathbb{N}}$ be a sequence of arbitrary subsets of $\mathbb{C}$. Define the limits inferior and superior of $\left\{E_{n}\right\}_{n \in \mathbb{N}}$, denoted respectively by $\lim \inf E_{n}$ and $\lim \sup E_{n}$, as follows:

- $\liminf E_{n}=\{\lambda \in \mathbb{C} \mid$ for every $\epsilon>0$, there exists $N \in \mathbb{N}$ such that $B(\lambda, \epsilon) \cap E_{n} \neq \emptyset$ for all $\left.n \geq N\right\}$.

- $\lim \sup E_{n}=\{\lambda \in \mathbb{C} \mid$ for every $\epsilon>0$, there exists $J \subseteq \mathbb{N}$ infinite such that $B(\lambda, \epsilon) \cap E_{n} \neq \emptyset$ for all $\left.n \in J\right\}$.

Next lemma is helpful to demonstrate several results in this paper.

Lemma 4.1. Let $\left\{E_{n}\right\}_{n \in \mathbb{N}}$ be a sequence of subsets of $\mathbb{C}$. The following statements are satisfied:

(a) $\liminf E_{n}$ and $\lim \sup E_{n}$ are closed subsets of $\mathbb{C}$.

(b) $\lambda \in \lim \sup E_{n}$ if and only if there exists an increasing sequence of natural numbers $n_{1}<n_{2}<n_{3}<\cdots$ and points $\lambda_{n_{k}} \in E_{n_{k}}$ for all $k \in \mathbb{N}$ such that $\lim \lambda_{n_{k}}=\lambda$. 
(c) $\lambda \in \liminf E_{n}$ if and only if there exists a sequence $\left\{\lambda_{n}\right\}_{n \in \mathbb{N}}$ in $\mathbb{C}$ such that $\lim \lambda_{n}=\lambda$, and for some $n_{0} \in \mathbb{N}, \lambda_{n} \in \overline{E_{n}}$ for all $n \geq n_{0}$.

Lemma 4.2. Let $\left\{E_{n}\right\}_{n \in \mathbb{N}}$ be a sequence of closed subsets of $\mathbb{C}$. If there exists $K$ a compact subset of $\mathbb{C}$ for which $E_{n} \subseteq K$ for all $n \in \mathbb{N}$. Then $E_{n} \rightarrow E$ in the Hausdorff metric if and only if $\lim \sup E_{n} \subseteq E$ and $E \subseteq \liminf E_{n}$.

Let $T_{n}, T \in B(X)$. We say that $T_{n}$ converge in norm to $T$, and is denoted by $T_{n} \rightarrow T$, if $\lim _{n \rightarrow \infty}\left\|T_{n}-T\right\|=0$. Consider $\varphi: B(X) \rightarrow \mathcal{S}$, this function is continuous at $T \in B(X)$ if and only if for each $\left\{T_{n}\right\}_{n \in \mathbb{N}}$ in $B(X)$ such that $T_{n} \rightarrow T$, it follows that $\varphi\left(T_{n}\right) \rightarrow \varphi(T)$, where the latter convergence is with respect to the Hausdorff metric.

Remark 4.3. In particular, if $\varphi \in\left\{\sigma, \sigma_{a p}, \sigma_{s u}\right\}$ and $T \in B(X)$. Then $\varphi$ : $B(X) \rightarrow \mathcal{S}$ is continuous at $T$ if and only if $\lim \sup \varphi\left(T_{n}\right) \subseteq \varphi(T)$ and $\varphi(T) \subseteq$ $\lim \inf \varphi\left(T_{n}\right)$ for all $T_{n} \rightarrow T$. It results from Lemma 4.2 , because if $T_{n} \rightarrow T$, then

$$
\varphi\left(T_{n}\right) \subseteq \sigma\left(T_{n}\right) \subseteq B\left(0,\left\|T_{n}\right\|\right) \subseteq B\left(0, \epsilon_{1}+\|T\|\right)
$$

for all $n \in \mathbb{N}$ and some $\epsilon_{1}>0$.

On the other hand, it also holds that if $\varphi \in\left\{\sigma, \sigma_{a p}, \sigma_{s u}\right\}$ and $T: X \rightarrow X$ is any bounded operator, then $\lim \sup \varphi\left(T_{n}\right) \subseteq \varphi(T)$ for all $T_{n} \rightarrow T$. This result is an immediate consequence of the following lemma due to Halmos and Lumer $[12]$.

Lemma 4.4. Let $\Omega$ be a closed subset of $B(X)$ and $\tau$ a function defined by

$$
\tau(T)=\{\lambda \in \mathbb{C} \mid \lambda I-T \in \Omega\}, \quad T \in B(X) .
$$

Then $\lim \sup \tau\left(T_{n}\right) \subseteq \tau(T)$ for all $T_{n} \rightarrow T$.

Proof. Consider $\lambda \in \limsup \tau\left(T_{n}\right)$ and suppose that $\lambda \notin \tau(T)$. Inasmuch as $\Omega$ is closed, there is $\epsilon>0$ such that $B(\lambda I-T, \epsilon) \subseteq B(X) \backslash \Omega$. Let $n_{1}<n_{2}<\cdots$ be an increasing sequence of natural numbers and let $\lambda_{n_{k}} \in \tau\left(T_{n_{k}}\right)$ for all $k \in \mathbb{N}$ such that $\lambda_{n_{k}} \rightarrow \lambda$. Since $T_{n} \rightarrow T$, there is $k_{0} \in \mathbb{N}$ such that for every $k \geq k_{0}, \lambda_{n_{k}} I-T_{n_{k}} \in B(\lambda I-T, \epsilon)$. Take $k \geq k_{0}$, then $\lambda_{n_{k}} I-T_{n_{k}} \notin \Omega$ and so $\lambda_{n_{k}} \notin \tau\left(T_{n_{k}}\right)$, this is a contradiction.

The set of bounded operators that are invertible, the set of all $T \in B(X)$ which are one-to-one and have $R(T)$ closed and the set of surjective bounded operators are open in $B(X)$ (see [5, Theorem 2.5.6]). So by Lemma 4.4 the following theorem is satisfied.

Theorem 4.5. Let $\varphi \in\left\{\sigma, \sigma_{a p}, \sigma_{s u}\right\}$. For each $T_{n} \rightarrow T$, it holds that

$$
\lim \sup \varphi\left(T_{n}\right) \subseteq \varphi(T) .
$$

However, in general the functions $\sigma, \sigma_{a p}$ and $\sigma_{s u}$ are not continuous as shown in the next example. 
Example 4.6. Let $U$ be the unilateral shift on $\ell^{2}(\mathbb{N})$ and let $T, T_{n}$, be operators defined on $\ell^{2}(\mathbb{N}) \oplus \ell^{2}(\mathbb{N})$ as

$$
T=\left[\begin{array}{cc}
U & 0 \\
0 & U^{*}
\end{array}\right] \quad T_{n}=\left[\begin{array}{cc}
U & \frac{1}{n}\left(I-U U^{*}\right) \\
0 & U^{*}
\end{array}\right] .
$$

Observe that $T_{n} \rightarrow T$, but $\sigma\left(T_{n}\right) \nrightarrow \rightarrow \sigma(T)$. Indeed, each $T_{n}$ is similar to $T_{1}$ and $T_{1}$ is an unitary operator, so for every $n, \sigma\left(T_{n}\right)=\sigma\left(T_{1}\right)=\{\lambda \in \mathbb{C}|| \lambda \mid=$ $1\}$, and $\sigma(T)=\{\lambda \in \mathbb{C}|| \lambda \mid \leq 1\}$.

From Theorem 4.5, in order to see that $\varphi: B(X) \rightarrow \mathcal{S}$ is continuous at $T \in B(X)$, where $\varphi \in\left\{\sigma, \sigma_{a p}, \sigma_{s u}\right\}$, it is sufficient to show that for each $\left\{T_{n}\right\}_{n \in \mathbb{N}}$ in $B(X)$ that converges to $T$, it is satisfied that $\tau(T) \subseteq \lim \inf \tau\left(T_{n}\right)$.

Next, let us note certain sets of $\sigma(T)$ for which are included in $\lim \inf \sigma\left(T_{n}\right)$. First, the isolated points of $\sigma(T)$ are included in $\lim \inf \tau\left(T_{n}\right)$.

Proposition 4.7 ([14]). If $T_{n}, T \in B(X)$ be such that $T_{n} \rightarrow T$, then iso $\sigma(T) \subseteq$ $\liminf \sigma\left(T_{n}\right)$, where iso $\sigma(T)=\{$ isolated points of $\sigma(T)\}$.

If $T \in B(X)$ and $\Lambda$ is a spectral set for $T$ (i.e., $\Lambda$ is both open and closed in $\sigma(T))$, define the corresponding spectral projection as

$$
P(T, \Lambda)=\frac{1}{2 \pi i} \int_{\mathcal{C}}(z I-T)^{-1} d z,
$$

where $\mathcal{C}$ is a Cauchy contour that separates $\Lambda$ from $\sigma(T) \backslash \Lambda$. For $T \in B(X)$ define

$$
\pi_{0}(T)=\{\lambda \in \text { iso } \sigma(T) \mid P(T,\{\lambda\}) \text { has finite rank }\} .
$$

Proposition 4.8. Let $T_{n}, T \in B(X)$ be such that $T_{n} \rightarrow T$. Then $\pi_{0}(T) \subseteq$ $\liminf \pi_{0}\left(T_{n}\right)$.

Proof. See [1, Corollary 2.13].

Next proposition give another collection of points that is included in lim $\inf \sigma\left(T_{n}\right)$.

Proposition 4.9. If $\left\{T_{n}\right\}$ is a sequence in $B(X)$ such that $T_{n} \rightarrow T$, then $\sigma(T) \backslash \sigma_{a p}(T) \subseteq \liminf \sigma\left(T_{n}\right)$.

Proof. Let $\lambda \in \sigma(T) \backslash \sigma_{a p}(T)$, then $\lambda I-T$ is an injective operator with closed range, this implies that $\lambda I-T$ is a semi-Fredholm operator and $i(\lambda I-T) \leq 0$, but $\lambda I-T$ is not invertible, so $i(\lambda I-T)<0$. Observe that, if there exists an increasing sequences of natural numbers $\left\{n_{k}\right\}_{k \in \mathbb{N}}$ such that $\lambda I-T_{n_{k}}$ is invertible for all $k \in \mathbb{N}$, then by the continuity of index, $i(\lambda I-T)=0$, that is a contradiction. So there exists $n_{0} \in \mathbb{N}$ such that $\lambda \in \sigma\left(T_{n}\right)$, for all $n \geq n_{0}$, which implies that $\lambda \in \liminf \sigma\left(T_{n}\right)$.

Theorem 4.10. If $\sigma_{a p}$ is continuous $T$, then $\sigma$ is continuous at $T$.

Conway and Morrel in their seminal paper [6] characterize the continuity of the spectrum in the algebra $B(H)$, where $H$ is a Hilbert space: 
Theorem 4.11 ([6, Teorema 3.1]). Let $H$ be a Hilbert space. The function $\sigma$ is continuous at $T \in B(H)$, if and only if, for each $\lambda \in \sigma(T) \backslash \overline{\rho_{s-F}^{ \pm}(T)}$ and $\epsilon>0$, the ball $B(\lambda, \epsilon)$ contains a component of $\sigma_{s-F}(T) \cup \pi_{0}(T)$.

Later, in [7], they give a characterization of continuity of the approximate point spectrum:

Theorem 4.12. Let $H$ be a Hilbert space. The function $\sigma_{a p}$ is continuous at $T \in B(H)$, if and only if,

(a) $\sigma$ is continuous at $T$,

(b) $\rho_{s-F}^{-}(T) \cap \sigma_{p}(T)=\emptyset$,

(c) $\rho_{s-F}^{-\infty}(T)=\operatorname{int} \overline{\rho_{s-F}^{-\infty}(T)}$ and,

(d) for every $-\infty<k \leq-1$, it holds that, for each $\lambda \in \operatorname{int} \overline{\rho_{s-F}^{k}(T)} \backslash \rho_{s-F}^{k}(T)$ and $\epsilon>0$, the ball $B(\lambda, \epsilon)$ contains a component of $\sigma_{s-F}(T)$.

In this paper we observe that for the sufficient condition in Theorem 4.12 the several hypotheses can be reduced on the class of bounded operators (now defined on a Banach space) whose adjoint has SVEP. On the other hand, it should be noted that Conway and Morrel show the above theorem using the following equivalence (see $[7$, p. 462]):

- $\sigma$ is continuous at $T \in B(H)$ if and only if int $\left(\sigma(T) \backslash \sigma_{W}(T)\right)=\emptyset$ and, for each $\lambda \in \sigma_{s-F}(T) \backslash \overline{\rho_{s-F}^{ \pm}(T)}$ and $\epsilon>0$, the ball $B(\lambda, \epsilon)$ contains a component of $\sigma_{s-F}(T)$.

But this proposition is false as shown in Example 4.13. However, the conclusion of above mentioned theorem is true and the proof is a slight modification of the given.

Example 4.13. Let $\alpha_{n k}=\left(1+\frac{1}{n}\right) \exp \left(2 \pi i \frac{k}{n}\right)$ for all $n \in \mathbb{N}$ and $1 \leq k \leq n$, and consider $M: \ell^{2}(\mathbb{N}) \rightarrow \ell^{2}(\mathbb{N})$ the diagonal operator defined by

$$
M=\left(\begin{array}{cccc}
\alpha_{11} & & & \\
& \alpha_{21} & & \\
& & \alpha_{22} & \\
& & & \ddots
\end{array}\right) .
$$

It is clear that $\sigma_{p}(M)=\left\{\alpha_{n k} \mid n \in \mathbb{N}, 1 \leq k \leq n\right\}$ where each eigenvalue has geometry multiplicity one. Let $\left\{\alpha_{m}\right\}_{m \in \mathbb{N}}$ be and indexation of $\left\{\alpha_{11}, \alpha_{21}, \alpha_{22}, \alpha_{31}, \alpha_{32}, \ldots\right\}$. It is not difficult to prove that

$$
\lambda \in \rho(M)=\mathbb{C} \backslash \sigma(M) \Leftrightarrow \inf _{m}\left|\lambda-\alpha_{m}\right|>0 .
$$

Thus

$$
\begin{aligned}
\sigma(M) & =\sigma_{p}(M) \cup\left\{\lambda \in \mathbb{C}\left|\inf _{m}\right| \lambda-\alpha_{m} \mid=0, \text { and for all } m, \lambda \neq \alpha_{m}\right\} \\
& =\left\{\alpha_{m}\right\}_{m \in \mathbb{N}} \cup\{\lambda \in \mathbb{C}|| \lambda \mid=1\} .
\end{aligned}
$$


Let $m \in \mathbb{N}$, since $\inf \left\{\left|\alpha_{m}-\alpha_{j}\right| \mid j \in \mathbb{N}\right.$ and $\left.j \neq m\right\}>0$ and for each $\left\{y_{j}\right\}_{j \in \mathbb{N}} \in \overline{R\left(\alpha_{m} I-M\right)}, y_{m}=0$. It follows that $R\left(\alpha_{m} I-M\right)$ is a closed subset of $\ell^{2}(\mathbb{N})$. Therefore, for every $m \in \mathbb{N}, \alpha_{m} I-M$ is a semi-Fredholm operator, and clearly $\alpha_{m}$ is an isolated point of $\sigma(M)$. Consequently $\left\{\alpha_{m}\right\}_{m \in \mathbb{N}} \subseteq \pi_{0}(M)$.

From that $\lambda I-M$ is injective for all $\lambda \notin\left\{\alpha_{m}\right\}_{m \in \mathbb{N}}$, it is easy to see that $\sigma_{s-F}(M)=\{\lambda \in \mathbb{C}|| \lambda \mid=1\}, \rho_{s-F}^{ \pm}(M)=\emptyset$ and $\pi_{0}(M)=\left\{\alpha_{m}\right\}_{m \in \mathbb{N}}$. This implies that

$$
\sigma(M)=\overline{\pi_{0}(M)} \subseteq \liminf \pi_{0}\left(M_{n}\right) \subseteq \liminf \sigma\left(M_{n}\right)
$$

for all $M_{n} \rightarrow M$, that is, $\sigma$ is continuous at $M$. However, for every $\lambda_{0} \in$ $\sigma_{s-F}(T) \backslash \overline{\rho_{s-F}^{ \pm}(T)}$ and $\epsilon>0$ such that $\{\lambda \in \mathbb{C}|| \lambda \mid=1\} \nsubseteq B\left(\lambda_{0}, \epsilon\right)$, the ball $B\left(\lambda_{0}, \epsilon\right)$ does not contain a component of $\sigma_{s-F}(T)$.

Lemma 4.14 ([6, Lemma 1.5]). Consider $\left\{T_{n}\right\}_{n \in \mathbb{N}}$ in $\mathcal{B}(X)$ such that $T_{n} \rightarrow T$.

(a) If $C$ is a component of $\sigma(T)$ and $U$ an open set containing $C$, then there exits $n_{0} \in \mathbb{N}$ such that for each $n \geq n_{0}$, $U$ contains a component of $\sigma\left(T_{n}\right)$.

(b) If $C$ is a component of $\sigma_{e}(T)$ and $U$ an open set containing $C$, then there exists $n_{0} \in \mathbb{N}$ such that for each $n \geq n_{0}$, $U$ contains a component of $\sigma_{e}\left(T_{n}\right)$.

Proof. The following proof works for any Banach algebra, so that only part (a) is proved.

Let $C$ be a component of $\sigma(T)$ and $U$ be an open set of $\mathbb{C}$ that contains to $C$. The set $\sigma(T) \backslash U$ is closed in $\sigma(T)$, and $C \cap[\sigma(T) \backslash U]=\emptyset$. Then there exists $X_{1} \neq \emptyset$ open and closed in $\sigma(T)$ such that $C \subseteq X_{1} \subseteq U$. Observe that $U \cap\left[\mathbb{C} \backslash\left(\sigma(T) \backslash X_{1}\right)\right]$ is open in $\mathbb{C}$ and $X_{1} \subseteq U \cap\left[\mathbb{C} \backslash\left(\sigma(T) \backslash X_{1}\right)\right]$, thus by $\left[1\right.$, Theorem 1.21], there exists a Cauchy domain $D$ such that $X_{1} \subseteq D \subseteq \bar{D} \subseteq$ $U \cap\left[\mathbb{C} \backslash\left(\sigma(T) \backslash X_{1}\right)\right]$. Let $\mathcal{C}$ be a Cauchy contour determined by the boundary of $D$. Then $\mathcal{C}$ separates $X_{1}$ from $\sigma(T) \backslash X_{1}$.

By $[1$, Proposition 2.9(a)], there exits $N \in \mathbb{N}$ such that for every $n \geq N, \mathcal{C}$ lies in $\rho\left(T_{n}\right), \Lambda_{n}=\sigma\left(T_{n}\right) \cap D$ is a spectral set for $T_{n}$, and $\mathcal{C}$ separates $\Lambda_{n}$ from $\sigma\left(T_{n}\right) \backslash \Lambda_{n}$. Moreover, if

$$
P\left(T_{n}, \Lambda_{n}\right)=\frac{1}{2 \pi i} \int_{\mathcal{C}}\left(z I-T_{n}\right)^{-1} d z, \quad P\left(T, X_{1}\right)=\frac{1}{2 \pi i} \int_{\mathcal{C}}\left(z I-T_{n}\right)^{-1} d z,
$$

then $P\left(T_{n}, \Lambda_{n}\right) \rightarrow P\left(T, X_{1}\right)$. Note $P\left(T, X_{1}\right) \neq 0$ because $X_{1} \neq \emptyset$ (see $[1$, Corollary 1.27]). Hence there exists $n_{0}>N$ such that $P\left(T_{n}, \Lambda_{n}\right) \neq 0$ for all $n \geq n_{0}$. Thus by [1, Corollary 1.27], $\Lambda_{n} \neq \emptyset$ for all $n \geq n_{0}$.

Let $n \geq n_{0}$ arbitrary. Since $\Lambda_{n}$ is both open and closed in $\sigma\left(T_{n}\right)$ and $\Lambda_{n} \neq \emptyset$, it follows that, there exists $C_{n}$ a component of $\sigma\left(T_{n}\right)$ such that $C_{n} \subseteq \Lambda_{n}$. Observe that $\Lambda_{n} \subseteq D \subseteq U$. Thus $C_{n} \subseteq U$. Therefore $U$ contains a component of $\sigma\left(T_{n}\right)$ for all $n \geq n_{0}$.

In order to study the continuity of $\sigma_{a p}$ on the clase of operators defined on Banach spaces we have introduced the sets $\phi_{+}(T), \phi_{-}(T), \phi_{ \pm \infty}(T)$ instead of 
the sets $\rho_{s-F}^{+}(T), \rho_{s-F}^{-}(T), \rho_{s-F}^{ \pm \infty}(T)$. The following lemma is a generalization of [7, Lemma 3.1] for the case of Banach spaces.

Lemma 4.15 ([15, Lemma 2]). If $T_{n} \rightarrow T$ in $\mathcal{B}(X)$ and $\lambda \notin \overline{\phi_{ \pm \infty}(T)}$ is such that, for every $\epsilon>0$, the ball $B(\lambda, \epsilon)$ contains a component of $\sigma_{\text {lre }}(T)$, then $\lambda \in \liminf \sigma_{s-F}\left(T_{n}\right)$.

Proof. Let $\epsilon>0$. Since $\lambda \notin \overline{\phi_{ \pm \infty}(T)}$, there is $r>0$ such that $B(\lambda, r) \cap$ $\overline{\phi_{ \pm \infty}(T)}=\emptyset$. Set $\epsilon_{1}=\min \{\epsilon, r\}$, by hypothesis, $B\left(\lambda, \epsilon_{1}\right)$ contains a component $C$ of $\sigma_{\text {lre }}(T)$, then $C \cap \overline{\phi_{ \pm \infty}(T)}=\emptyset$ and so by Theorem 3.4, $C$ is a component of $\sigma_{e}(T)$. From Lemma 4.14(b), there exists $n_{0} \in \mathbb{N}$, such that for every $n \geq n_{0}$, $B\left(\lambda, \epsilon_{1}\right)$ contains a component $C_{n}$ of $\sigma_{e}\left(T_{n}\right)$. By this and Proposition 3.3(c), it follows that for each $n \geq n_{0}$,

$$
\emptyset \neq \partial C_{n} \subseteq \partial \sigma_{e}\left(T_{n}\right) \subseteq \sigma_{s-F}\left(T_{n}\right) .
$$

Thus for every $n \geq n_{0}, B(\lambda, \epsilon) \cap \sigma_{s-F}\left(T_{n}\right) \neq \emptyset$, which implies that $\lambda \in$ $\liminf \sigma_{s-F}\left(T_{n}\right)$.

We recall that an operator $T \in \mathcal{B}(X)$ is said to have the single-valued extension property at $\lambda \in \mathbb{C}$, abbreviate $T$ has SVEP at $\lambda \in \mathbb{C}$, if for every neighborhood $U_{\lambda}$ of $\lambda$, the only analytic function $f: U_{\lambda} \rightarrow X$ which satisfies the equation $(\mu I-T) f(\mu)=0$ for all $\mu \in U_{\lambda}$ is the function $f \equiv 0$.

It is clear that $T$ has SVEP at every point of the resolvent $\rho(T)$, moreover from identity theorem for analytic functions, both $T$ and $T^{*}$ has SVEP at every point of the boundary $\partial \sigma(T)$ of the spectrum. In particular, both $T$ and $T^{*}$ have SVEP at every isolated point of the spectrum.

Following [13], we say that $T \in B(X)$ satisfies the Browder's theorem, if $\sigma(T) \backslash \sigma_{W}(T)=\pi_{0}(T)$. This property is characterized in terms of the SVEP. Indeed, a necessary and sufficient condition for $T$ satisfies Browder's theorem is that $T$ has SVEP at every point $\lambda \notin \sigma_{W}(T)$.

Theorem 4.16 ([15, Theorem 3]). Let $T \in \mathcal{B}(X)$ such that $T^{*}$ has SVEP at every $\beta \notin \sigma_{\text {lre }}(T)$. If for each $\lambda \in \sigma_{\text {lre }}(T) \backslash \overline{\phi_{+}(T)}$ and $\epsilon>0$, the ball $B(\lambda, \epsilon)$ contains a component of $\sigma_{\text {lre }}(T)$, then $\sigma_{\text {ap }}$ is continuous at $T$.

Proof. Let $\left\{T_{n}\right\}_{n \in \mathbb{N}}$ be a sequence in $\mathcal{B}(X)$ that converges at $T$, and let $\lambda \in$ $\sigma_{a p}(T)$.

Case I: $\lambda \notin \sigma_{\text {lre }}(T)$.

In this case $\lambda I-T$ is a semi-Fredholm operator and $T^{*}$ has SVEP at $\lambda$, so by $\left[2\right.$, Corollary 3.19], $i(\lambda I-T) \geq 0$. Suppose that $i(\lambda I-T)=0$. Since $T^{*}$ has SVEP at every $\beta \notin \sigma_{w}(T)$, it follows that $T^{*}$ satisfies Browder's theorem, and consequently, $T$ satisfies too. Thus $\lambda \in \sigma(T) \backslash \sigma_{w}(T)=\pi_{0}(T)$. Consequently by Proposition 4.8, $\lambda \in \liminf \pi_{0}\left(T_{n}\right) \subseteq \liminf \sigma_{a p}\left(T_{n}\right)$

Now, suppose that $i(\lambda I-T)>0$. If $\lambda \notin \liminf \sigma_{a p}\left(T_{n}\right)$, then there exist $\epsilon_{1}>0$ and a increasing sequence of natural numbers $n_{1}<n_{2}<n_{3}<\cdots$ such that $B\left(\lambda, \epsilon_{1}\right) \cap \sigma_{a p}\left(T_{n_{k}}\right)=\emptyset$ for all $k \in \mathbb{N}$. Since $\lambda \notin \sigma_{a p}\left(T_{n_{k}}\right)$, it follows that 
$\lambda I-T_{n_{k}}$ is an injective operator with close range. This implies that $\lambda I-T_{n_{k}} \in$ $\Phi_{+}(X)$ and $i\left(\lambda I-T_{n_{k}}\right) \leq 0$. On the other hand, $\lambda I-T_{n_{k}} \rightarrow \lambda I-T$, thus by continuity of index, it follows that $i(\lambda I-T) \leq 0$ which is a contradiction. That proves $\lambda \in \liminf \sigma_{a p}\left(T_{n}\right)$.

Case II: $\lambda \in \sigma_{\text {lre }}(T)$.

By the proof of Case I, we have that $\phi_{+}(T) \subseteq \liminf \sigma_{a p}\left(T_{n}\right)$. Thus $\overline{\phi_{+}(T)} \subseteq$ $\liminf \sigma_{a p}\left(T_{n}\right)$ because $\liminf \sigma_{a p}\left(T_{n}\right)$ is a closed set. Therefore if $\lambda \in \overline{\phi_{+}(T)}$, then $\lambda \in \liminf \sigma_{a p}\left(T_{n}\right)$.

Let $\lambda \notin \overline{\phi_{+}(T)}$. By the hypothesis and Lemma 4.15 (note that $\phi_{-\infty}(T)=\emptyset$ ), we get that $\lambda \in \liminf \sigma_{s-F}\left(T_{n}\right)$. But since $\sigma_{s-F}\left(T_{n}\right) \subseteq \sigma_{a p}\left(T_{n}\right)$ for all $n \in \mathbb{N}$, it follows that $\lambda \in \liminf \sigma_{a p}\left(T_{n}\right)$. Therefore $\sigma_{a p}$ is continuous at $T$.

Corollary 4.17. If $T \in B(X)$ satisfies the hypothesis of Theorem 4.16, then $\sigma$ is continuous at $T$.

Proof. It follows by Theorem 4.16 and Theorem 4.10 .

Example 4.18. The unilateral shift $U: \ell^{2}(\mathbb{N}) \rightarrow \ell^{2}(\mathbb{N})$ defined by

$$
U(x)=\left(0, x_{1}, x_{2}, x_{3}, \ldots\right), \quad x=\left(x_{1}, x_{2}, x_{3}, \ldots\right),
$$

is a continuity point of $\sigma$.

In Theorem 4.16 the set of points $\lambda$ for which the ball $B(\lambda, \epsilon)$ contains a component of $\sigma_{\text {lre }}(T)$ for all $\epsilon>0$ may be reduced. Indeed:

Theorem 4.19. Let $T \in \mathcal{B}(X)$ such that $T^{*}$ has $S V E P$ at every $\beta \notin \sigma_{\text {lre }}(T)$. If for each $\lambda \in \sigma_{\text {lre }}(T) \backslash \overline{\phi_{+}(T) \cup \pi_{0}(T)}$ and $\epsilon>0$, the ball $B(\lambda, \epsilon)$ contains a component of $\sigma_{\text {lre }}(T)$, then $\sigma_{a p}$ is continuous at $T$.

As immediate consequence of Theorem 4.16 and some duality results, we obtain sufficient conditions for continuity of $\sigma_{s u}$.

Proposition 4.20. If $\sigma_{a p}$ is continuous at $T^{*}$, then $\sigma_{s u}$ is continuous at $T$.

Proof. Let $\left\{T_{n}\right\}_{n \in \mathbb{N}}$ be a sequence in $B(X)$ such that $T_{n} \rightarrow T$, then $T_{n}^{*} \rightarrow T^{*}$ and so $\sigma_{a p}\left(T_{n}^{*}\right) \rightarrow \sigma_{a p}\left(T^{*}\right)$. Since $\sigma_{a p}\left(L^{*}\right)=\sigma_{s u}(L)$ for every operator $L$, it follows that $\sigma_{s u}\left(T_{n}\right) \rightarrow \sigma_{s u}(T)$.

Corollary 4.21. Let $T \in \mathcal{B}(X)$ such that $T$ has $S V E P$ at every $\beta \notin \sigma_{\text {lre }}(T)$. If for each $\lambda \in \sigma_{\text {lre }}(T) \backslash \overline{\phi_{-}(T)}$ and $\epsilon>0$, the ball $B(\lambda, \epsilon)$ contains a component of $\sigma_{\text {lre }}(T)$, then $\sigma_{\text {su }}$ is continuous at $T$.

Proof. In order to prove this corollary we use Theorem 4.16. First observe that $\sigma_{a p}\left(T^{*}\right)=\sigma_{s u}(T), \sigma_{\text {lre }}\left(T^{*}\right)=\sigma_{\text {lre }}(T)$ and $\phi_{-}(T)=\phi_{+}\left(T^{*}\right)$. Take $\beta \notin$ $\sigma_{\text {lre }}\left(T^{*}\right)$, then $\beta I-T$ is a semi-Fredholm operator and $T$ has SVEP at $\beta$, this implies, by [2, Theorem 3.23], that $\beta$ is not a cluster point of $\sigma_{a p}(T)$. But, since $\sigma_{a p}(T)=\sigma_{s u}\left(T^{*}\right)=\sigma_{a p}\left(T^{* *}\right)$, it follows that $\beta$ is not a cluster point of $\sigma_{a p}\left(T^{* *}\right)$. Again, from [2, Theorem 3.23], $T^{* *}$ has SVEP at $\beta$. 
Consequently, $T^{* *}$ has SVEP at every point $\beta \notin \sigma_{\text {lre }}\left(T^{*}\right)$ and its clear that the other hypotheses of Theorem 4.16 are satisfied. Thus $\sigma_{a p}$ is continuous at $T^{*}$ and, by Proposition $4.20, \sigma_{s u}$ is continuous at $T$.

Acknowledgments. This work was supported in part by PROMEP (Apoyo del cuerpo académico UTMIX-CA-33).

\section{References}

[1] M. Ahues, A. Largillier, and B. V. Limaye, Spectral Computations for Bounded Operators, Chapman \& Hall/CRC, 2001.

[2] P. Aiena, Fredholm and Local Spectral Theory with Applications to Multipliers, Kluwer Acad., 2004.

[3] C. Apostol, L. A. Fialkow, D. A. Herrero, and D. Voiculescu, Approximation of Hilbert space operators. Vol. II, Res. Notes Math. 102, Pitman, Boston, 1984.

[4] L. Burlando, Continuity of spectrum and spectral radius in algebras of operators, Ann. Fac. Sci. Toulouse Math. 9 (1988), no. 1, 5-54.

[5] S. R. Caradus, W. E. Pfaffenberger, and B. Yood, Calkin Algebras and Algebras of Operators on Banach Spaces, Marcel Dekker, 1974.

[6] J. B. Conway and B. B. Morrel, Operators that are points of spectral continuity, Integral Equations Operator Theory 2 (1979), no. 2, 174-198.

[7] _ Operators that are points of spectral continuity. II, Integral Equations Operator Theory 4 (1981), no. 4, 459-503.

[8] S. V. Djordjević, The continuity of the essential approximative point spectrum, Facta Univ. Ser. Math. Inform. 10 (1995), 97-104.

[9] S. V. Djordjević and Y. M. Han, Browder's theorems and spectral continuity, Glasg. Math. J. 42 (2000), no. 3, 479-486.

[10] $\longrightarrow$, Operator matrices and spectral continuity, Glasg. Math. J. 43 (2001), no. 3, 487-490.

[11] B. P. Duggal, I. H. Jeon, and I. H. Kim, Continuity of the spectrum on a class of upper triangular operator matrices, J. Math. Anal. Appl. 370 (2010), 584-587.

[12] P. R. Halmos and G. Lumer, Square roots of operators. II, Proc. Amer. Math. Soc. 5 (1954), 589-595.

[13] R. E. Harte and W. Y. Lee, Another note on Weyl's theorem, Trans. Amer. Math. Soc. 349 (1997), no. 5, 2115-2124.

[14] J. D. Newburgh, The variation of Spectra, Duke Math. J. 18 (1951), 165-176.

[15] S. Sánchez-Perales and S. V. Djordjević, Continuity of spectrum and approximate point spectrum on operator matrices, J. Math. Anal. Appl. 378 (2011), no. 1, 289-294.

Salvador SÁnchez-Perales

Universidad Tecnológica de La Mixteca

InSTITUTO DE Física Y MATEMÁTICAS

Km. 2.5 Carretera a Acatlima, 69000 Oaxaca, Mexico

E-mail address: es21254@yahoo.com.mx

Victor A. Cruz-Barriguete

Universidad Tecnológica de la Mixteca

Instituto DE Física y MATEMÁticas

Km. 2.5 Carretera a Acatlima, 69000 Oaxaca, Mexico

E-mail address: victorcruz@mixteco.utm.mx 